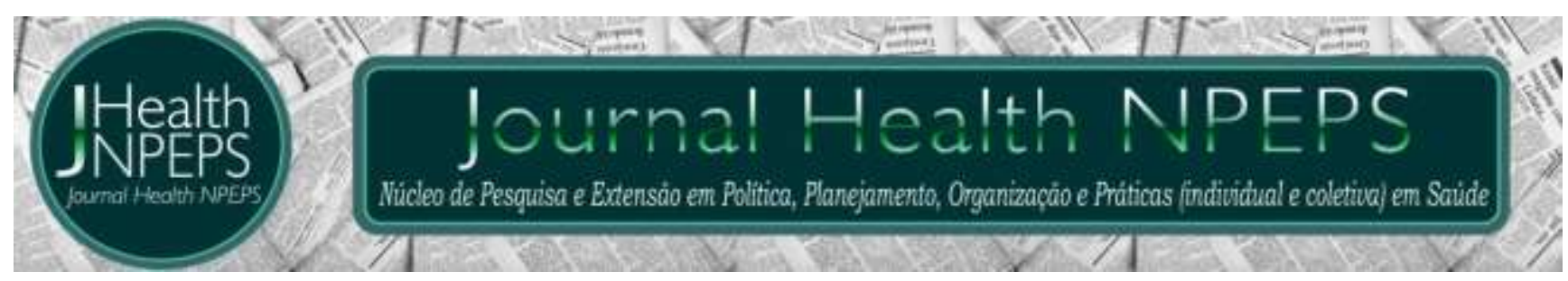

http://dx.doi.org/10.30681/252610103356

ARTIGO ORIGINAL

\title{
Sistematização da assistência de enfermagem na perspectiva do docente
}

\author{
Systematization of nursing assistance from the perspective of the teacher
}

Sistematización de la atención de enfermería en la perspectiva del docente

\author{
Micheline Midori Suzuki da Rocha ${ }^{1}$, Karina Nonato Mocheuti ${ }^{2}$, \\ Grasiela Cristina Silva Botelho Silvestre ${ }^{3}$, Claudia Moreira de Lima ${ }^{4}$, \\ Antonia Dinágila do Nascimento Ribeiro ${ }^{5}$
}

\section{RESUMO}

Objetivo: identificar como os docentes se preparam para ensinar a sistematização da assistência de enfermagem. Método: trata-se de um estudo exploratório, descritivo e com abordagem qualitativa, realizado em 2017, em uma instituição pública de ensino superior. Os dados foram coletados utilizando-se de entrevista semiestruturada e analisados pela análise do conteúdo. Resultados: o ensino da a sistematização da assistência de enfermagem provém desde a formação do professor até sua aplicabilidade em sala de aula. Os participantes do estudo consideram um desafio para esse ensino, em virtude do envolvimento de instituições de saúde para aplicabilidade desse aprendizado e muitas vezes, essas instituições inviabilizam a realização. Além disso, muitos discentes percebem esse conteúdo apenas como atividade extra, sem importância. 0 contato de muitos professores com esse conteúdo ocorre somente durante a graduação, o que dificulta posteriormente o ensino. Esses fatores prejudicam a implementação da sistematização da assistência de enfermagem tanto no contexto acadêmico como futuramente, no trabalho do enfermeiro. Considerações finais: conclui-se que durante a graduação as competências que regem este conteúdo são ensinadas em aulas teóricas e colocadas em práticas durante o estágio, porém há fragilidades no caminho que impossibilitam a consolidação do significado da a sistematização da assistência de enfermagem para o profissional.

Descritores: Diagnóstico de Enfermagem; Docentes de Enfermagem; Educação em Enfermagem.

\section{ABSTRACT}

Objective: to identify how teachers prepare to teach the systematization of nursing care. Method: this is an exploratory, descriptive study with a qualitative approach, carried out in 2017, in a public institution of higher education. Data were collected using a semistructured interview and analyzed by content analysis. Results: the teaching of the

${ }^{1}$ Enfermeira. Pós-graduanda em Gestão Pública pela Universidade Aberta do Brasil. Universidade do Estado de Mato Grosso (UNEMAT). Diamantino/MT, Brasil. E-mail: micheline suzuki@hotmail.com ORCID ID: http://orcid.org/0000-00032835-1542 Autor principal - Endereço para correspondência: Rua Para, 1019w, centro, Nova Olímpia/MT. CEP: 78.370.000.

${ }^{2}$ Enfermeira. Mestra em Educação. Diretora da Faculdade de Ciências da Saúde. Universidade do Estado de Mato Grosso (UNEMAT). Diamantino, Mato Grosso, Brasil. E-mail: enfkarinanonato@gmail.com ORCID ID: http://orcid.org/0000-0002$\underline{1800-2640}$

${ }^{3}$ Enfermeira. Mestra em Ambiente e Saúde. Docente da Graduação do Curso de Bacharelado em Enfermagem. Universidade do Estado de Mato Grosso - UNEMAT. Diamantino, Mato Grosso, Brasil. E-mail: enf.grasielabotelho@gmai.com ORCID ID: http://orcid.org/0000-0001-5367-4648

${ }^{4}$ Enfermeira. Mestra em Ambiente e Saúde. Docente da Graduação do Curso de Bacharelado em Enfermagem. Universidade do Estado de Mato Grosso - UNEMAT. Diamantino, Mato Grosso, Brasil. E-mail: cml claudiamoreira@hotmail.com ORCID ID: http://orcid.org/0000-0001-9864-7651

${ }^{5}$ Enfermeira. Graduada. Docente da Graduação do Curso de Bacharelado em Enfermagem. Universidade do Estado de Mato Grosso - UNEMAT. Diamantino, Mato Grosso, Brasil. E-mail: dinagilaribeiro@hotmail.com ORCID ID: http://orcid.org/0000-0001-7852-9199

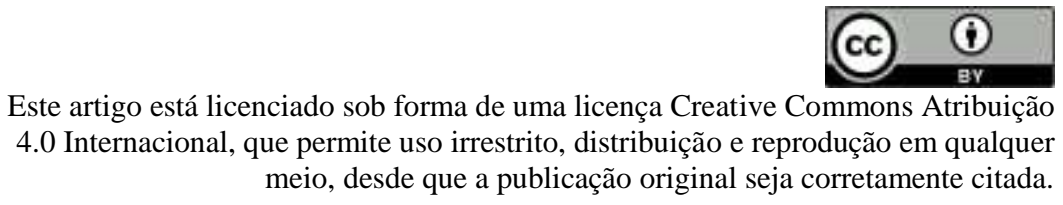


systematization of nursing care comes from the training of the teacher to its applicability in the classroom. The study participants consider this teaching a challenge, due to the involvement of health institutions in the applicability of this learning, and these institutions often make it unfeasible. In addition, many students perceive this content only as an extra and unimportant activity. The contact of many teachers with this content occurs only during graduation, which subsequently hampers teaching. These factors undermine the implementation of the systematization of nursing care both in the academic context and in the future, in the nurse's work. Conclusion: it is concluded that during graduation the competences that govern this content are taught in theoretical classes and placed in practices during the internship, but there are weaknesses in the path that make it impossible to consolidate the meaning of the systematization of nursing care for the professional.

Descriptors: Nursery Diagnosis; Faculty, Nursing; Education Nursing.

\section{RESUMEN}

Objetivo: identificar cómo los docentes se preparan para enseñar la sistematización de la asistencia de enfermería. Método: se trata de un estudio exploratorio, descriptivo y con abordaje cualitativo, realizado en 2017, en una institución pública de enseñanza superior. Los datos fueron recolectados utilizando entrevistas semiestructuradas y analizadas por el análisis del contenido. Resultados: la enseñanza de la sistematización de la asistencia de enfermería proviene desde la formación del profesor hasta su aplicabilidad en el aula. Los participantes del estudio consideran un desafío para esa enseñanza, en virtud de la participación de instituciones de salud para la aplicabilidad de ese aprendizaje y muchas veces, esas instituciones inviabilizan la realización. Además, muchos estudiantes perciben ese contenido sólo como actividad extra, sin importancia. El contacto de muchos profesores con ese contenido ocurre solamente durante la graduación, lo que dificulta posteriormente la enseñanza. Estos factores perjudican la implementación de la sistematización de la asistencia de enfermería tanto en el contexto académico, como en el futuro, en el trabajo del enfermero. Conclusión: se concluye que durante la graduación las competencias que rigen este contenido son enseñadas en clases teóricas y colocadas en prácticas durante la etapa, pero hay fragilidades en el camino que imposibilitan la consolidación del significado de la sistematización de la asistencia de enfermería para el profesional.

Descriptores: Diagnóstico de Enfermeira; Docentes de Enfermeira; Educación em Enfermería.

\section{INTRODUÇÃO}

A Sistematização da Assistência de Enfermagem (SAE) implantada no Brasil entre as décadas de 70-80 sob influência do trabalho de Wanda de Aguiar Horta, tem suas teorias fundamentadas nas necessidades humanas básicas. Desde seu surgimento passou por modificações em sua estrutura organizacional, estando hodiernamente em sua terceira geração. Está organizada em cinco fases que compreende: investigação, diagnóstico de enfermagem, planejamento de enfermagem, implementação e avaliação, com todas as etapas inter-relacionadas, interdependentes e recorrentes ${ }^{1,2}$.
A SAE é uma atividade privativa da enfermagem ${ }^{3-5}$, e tida como obrigatória sua implantação em toda instituição de saúde pública e/ou privada, que ofereça cuidados de enfermagem ${ }^{1}$. É um instrumento que permite a organização do trabalho quanto ao seu método, recursos humanos e instrumentos, mediante a implementação do Processo de Enfermagem $(\mathrm{PE})^{1,2}$.

Diante ao aludido se faz
necessário que o profissional que atua no processo do ensino no cuidar, acompanhe esse progresso, onde o ensino a base para aquisição do conhecimento, levando em consideração a cientificidade das ações, ultrapassando barreiras impostas pela 
desagregação do que se ensina na teoria com o que se vivencia na prática, ${ }^{6-8}$.

Destarte, a graduação tem a missão de proporcionar meios que viabilizem o conhecimento necessário ao aluno, este caracterizado por um perfil profissional com habilidades cognitivas e operacionais, sustentadas pela ética e comprometimento, para tanto se faz imprescindível que a SAE seja trabalhada diariamente nesse contexto, assim possibilitando o desenvolvimento de habilidades e atitudes em âmbito teórico e prático, contribuindo para a construção de uma enfermagem reflexiva, dinâmica e autônoma, exigindo sua aplicação de cuidados, com capacidades técnicas, intelectuais, cognitivas

e interpessoais ${ }^{7,9,10}$.

Entretanto, ainda há desafios no que se refere ao processo ensinoaprendizagem da SAE, se faz essencial um quadro de docentes capacitados para transmissão do conhecimento teórico e prático, bem como campos de estágios (práticas assistenciais) que ofertem tanto para o docente quanto aos discentes elementos para efetivarem a sua execução ${ }^{8,11,12}$.

Por fim, é necessário avaliar se estes elementos são suficientes para suprir as demandas durante o ensino da enfermagem tanto de forma teórica quanto prática, além da atuação do docente com relação aos conteúdos abordados durante o ensinar ${ }^{8,12}$.

Diante do exposto este trabalho buscou identificar como os docentes se preparam para ensinar a Sistematização da Assistência de Enfermagem (SAE).

\section{MÉTODO}

Estudo exploratório, descritivo e com delineamento qualitativo, realizado em um dos campus da Universidade do Estado de Mato Grosso (UNEMAT), no município de Diamantino-MT, Brasil.

Os participantes deste estudo foram enfermeiros docentes que faziam parte do quadro profissional da instituição no ano de 2017, e que concordaram em participar do estudo através da aceitação e assinatura do Termo de Consentimento Livre e Esclarecido (TCLE), por meio do qual foram apresentados os objetivos da pesquisa, a relevância da adesão dos mesmos na investigação e a garantia do anonimato, todas as respostas dos depoentes foram codificados pela abreviação de professor (prof), com as respectivas numerações subsequentes (prof1-prof8).

A coleta de dados foi realizada entre os meses de janeiro a março de 2017, por meio de entrevista norteada por instrumento elaborado pelos autores contendo questionário semiestruturado. Foram entrevistados um total de oito professores por meio de um questionário com auxílio de um gravador digital, em seguida as falas foram ouvidas $e$ transcritas de forma integral. A cada entrevista efetivada, procedemos à análise de conteúdo, agrupando-as informações e codificando-as a partir da interpretação das respostas, e quando novas ideias não eram acrescidas encerrou-se a coleta de dados, fazendo assim o uso da técnica de saturação.

Para a análise dos dados, com as informações obtidas, iniciou-se a préanálise, os objetivos iniciais foram retomados e alguns indicadores que 
permeiam a interpretação final, elaborados. Como a leitura flutuante e a constituição de corpus, que buscam descobrir os núcleos de sentido compostos nas transcrições, observando a presença e frequência de unidades de significação, com atenção para valores de referência e modelos de comportamento presentes ou subjacentes no discurso ${ }^{13}$.

A análise dos dados gerou quatro categorias: Aplicabilidade da SAE durante a graduação de enfermagem, conhecimento docente sobre SAE, ensino da SAE durante a graduação e a SAE e o futuro profissional.

Esta pesquisa foi desenvolvida após aprovação pelo Comitê de Ética em Pesquisa da UNEMAT, sob o Certificado de Apresentação para apreciação Ética (CAAE), número 62430216.6.0000.5166. 0 presente estudo obedeceu às normas de pesquisa com seres humanos, segundo a Resolução $n^{\circ}$ 466/2012 do Conselho Nacional de Saúde.

\section{RESULTADOS}

Fizeram parte da pesquisa oito docentes enfermeiros, dos quais, cinco possuem especialização e os demais mestrado. Em relação ao tempo de formação dos participantes, houve variação entre dois e 18 anos. Todos os participantes se encontravam lotados em disciplinas que vão do $4^{\circ}$ ao $8^{\circ}$ semestre.

\section{Aplicabilidade da SAE durante a graduação de enfermagem}

0 ensino da SAE durante a formação acadêmica é apontado como indispensável pelos depoentes, porém também são elencados fatores que dificultam o ensino e a aprendizagem desse método, como a desconversa da teoria com a prática ou até mesmo a impossibilidade de implantação do instrumento durante a graduação, como observado nos relatos:

\section{[...] a SAE é realizada de acordo com o atendimento ao paciente ou conforme a oportunidade em que temos, onde por muitas vezes não são oportunizadas por completo [...]. (Prof1) \\ No atendimento da Estratégia da Saúde da Família e também a realização da SAE que o aluno tem que entregar como requisito para nota. (Prof5)}

Diante ao aludido, é evidente a importância da associação entre teoria e prática durante a formação acadêmica de enfermagem, o que pode ser reforçado nas falas a seguir:

Consegue-se fazer a transposição da teoria com a prática e prática com a teoria. Na realidade quando se trabalha com a docência e na assistência consegue fazer a relação pedagógica com a prática do trabalho tentando deixar isso menos destoado possível, ou seja, sempre deixar tudo bem claro [...]. (Prof3)

[...] hoje na docência há uma perspectiva mais complexa que vem para questionar na condição de compreender e articular o empírico (realidade) e o teórico, que chamamos de práxis [...]. (Prof8)

\section{Conhecimento docente sobre SAE}

A conduta e as decisões tomadas durante o processo de ensino referem-se em suma ao conhecimento que os docentes possuem e transmitem, assegurando-lhes o agir certo e adequado ao ensinar, diante disso sempre estão à procura de estratégias para proporcionar aos seus alunos um ensino de qualidade.

Eu me preocupo com a qualidade do ensino em que estou passando para os alunos e assim sempre estou buscando a leitura de referências atualizadas acerca da temática, e vou buscar mais capacitações para aprimorar meus 
conhecimentos referente ao assunto, SAE. (Prof1)

[...] ]sempre estou buscando a leitura de referências atualizadas acerca da temática, e gostaria de estar realizando cursos acerca desse assunto que é tão importante para o ensino, já que é uma atividade privativa do enfermeiro. (Prof7)

Estou sempre revisando e acompanhando as publicações de NANDA, conhecimentos anatômicos $e$ fisiológicos, o processo de enfermagem, cuidados de enfermagem etc. (Prof8)

A preocupação docente não se abstém apenas ao seu conhecimento, mostram preocupação em estimular a busca por conhecimento em outras fontes além da fornecida em aula seja esta teórica e/ou prática.

Digo sempre, 'a biblioteca é a extensão da nossa sala de aula', desde o princípio da disciplina os discentes são estimulados a buscar e construir o próprio conhecimento, conforme dito acima. (Prof2)

\section{Ensino da SAE durante a graduação}

No processo de ensinoaprendizagem, o aluno se torna corresponsável pelo seu próprio aprendizado, de forma tradicional o papel do professor permanece ainda como elemento central, ou seja, mediador deste processo auxiliando o aluno quanto a aplicabilidade da SAE estimulando 0 mesmo a construir seu próprio conhecimento.

A metodologia é de acordo com a que é proposta pela instituição à qual se baseia na Teoria das Necessidades Humanas Básicas de Wanda Aguiar Horta e na prática nos embasamos através de um instrumento de coleta de dados e exame físico e com o apoio do NANDA para a confecção dos diagnósticos de enfermagem. (Prof1)

[...] utilizamos o pressuposto pautado na perspectiva de Paulo Freire, denominada de aprendizagemsignificativa, ou seja, pactuamos desde o início da disciplina a forma de trabalho: construção coletiva, a metodologia de ação: Roda de Conversa e Avaliação: consenso coletivo. (Prof2)

As entrevistas possibilitaram identificar que cada professor segue um caminho entre as diversas áreas de especializações existentes, onde a SAE envolve direta ou indiretamente a disciplina que estes ministram.

[...] envolvo a SAE específica a aquele cuidado em si, como por ex. saúde da mulher, então a SAE específico ao exame obstétrico e ginecológico [...]. (Prof4)

Atualmente a minha disciplina incorpora as Necessidades Humanas básicas, proporcionando os primeiros contatos do aluno com a SAE. (Prof7)

Trabalho a SAE no atendimento à mulher seja nos períodos de atenção à saúde ginecológica, pré-natal e puerperal e também na atenção aos cuidados imediatos ao neonato. (Prof8)

\section{A SAE e o futuro profissional}

A análise dessa categoria aponta para um construir do conhecimento não limitante ao que o professor explana, mas estar sempre buscando aprimorar o conhecimento, pois se permanecer somente no dizer do professor resultará em uma construção mecanicista, tornando-se um conhecimento fragmentado. Essa preocupação fica evidente nas falas a seguir:

Eu vejo que os alunos hoje em dia que estão se graduando eles não têm essa visão da importância que é sistematizar. [...] minha preocupação é de quem está se formando[...]. (Prof4)

É nítida a dificuldade que os acadêmicos têm quando o assunto é SAE, principalmente porque não conseguem associar as necessidades do paciente a uma sequência de cuidados, muitos alunos acham que tem que fazer o exame físico $e$ pronto. A fragmentação é perceptível. (Prof6) 


\section{DISCUSSÃO}

0 resultado da pesquisa denota que o ensino da SAE durante a graduação deve acontecer de forma ininterrupta dentre os semestres de ensino, possibilitando aos alunos uma associação da teoria com a prática desde o início de sua formação ${ }^{14,15}$. Entretanto o que acontece é ainda um ensino de forma descontinuada e fragmentada durante a graduação de enfermagem.

Os relatos permitem observar ainda que ensinar a SAE envolve diversos fatores que não se limitam ao profissional, mais inclui questões burocráticas administrativas imposta pela instituição de saúde onde é realizado o ensino da prática, desfavorecendo o ensino na sua integralidade. Em suma a SAE muitas vezes não é concretizada na sua integralidade, por falta de oportunidades que a instituição de saúde oferece desfavorecendo assim, o ensino?.

Todavia a consolidação do processo de enfermagem depende de vários fatores, dentre os quais se destaca - conhecimento prévio acerca da aplicabilidade da SAE e a forma como o profissional se apropria desse saber ${ }^{16}$. A busca por conhecimento é essencial na transmissão do mesmo, estando este interagindo na graduação entre docentediscente, como elemento indispensável para proporcionar mudanças no processo ensino-aprendizagem, favorecendo a construção de conhecimento coletivo ${ }^{8}$. Entretanto é essencial que se atualize constantemente os conhecimentos que relacionem a temática, levando-se em conta o enfoque interdisciplinar e multidimensional.

Portanto é necessário que o docente se aproprie do seu papel de mediador no processo de ensinoaprendizagem, no qual o aluno deve superar suas expectativas no que diz respeito ao conhecimento, aos questionamentos, a articulação do mundo com um recente método de educar ${ }^{17}$.

Destaca-se nos relatos a importância em incorporar novas formas de transmitir aprendizado, através de uma didática diferenciada com uso de tecnologias no processo de aprendizagem do aluno estimulando o processo ensinoaprendizagem, possibilitando a adesão de habilidades e conhecimentos essenciais a prática profissional, estimulando 0 pensamento crítico-reflexivo na tomada de decisão, contextualizando os saberes e desenvolvendo consciência diante do cenário ${ }^{18,19}$.

Durante as atividades práticas os docentes necessitam estar atentos quanto ao método educacional, no qual é preciso organizar e quando necessário oportunizar mudanças de pensamentos, ações e condutas. Isso é resultado de um planejamento ligado ao tipo de atividades, conteúdos, recursos disponíveis e da forma metodológica a ser aplicada durante o ensino, auxiliando o aluno na aplicabilidade da SAE, estimulando-o a construir seu próprio conhecimento ${ }^{8}$. Ressalta-se que ao construir esse conhecimento mútuo é essencial obter uma postura pedagógica dialética, com vistas a enriquecer a construção do conhecimento sem que o docente precise limitar ou impor seus dizeres. 
No processo de ensinoaprendizagem hodiernamente o aluno é corresponsável pelo seu próprio aprendizado, porém, o docente permanece como elemento essencial neste processo, atuando da melhor maneira para ensinar e aprender ${ }^{20}$. Esse espaço conquistado pelo estudante de enfermagem nos cenários da prática profissional durante a graduação não deve limitar-se ao conteúdo que o professor explana, mas estar em constante busca por conhecimento, a fim de evitar um aprendizado fragmentado.

As Diretrizes Curriculares Nacionais do Curso de Enfermagem vislumbram uma formação articulado com a prática profissional buscando uma atuação de forma reflexiva transformando a realidade, com auxílio de métodos de ensino e aprendizagem em cenários diversos tanto na teoria quanto na prática, possibilitando ao aluno ser protagonista do processo, permitindo o desenvolvimento de competências profissionais ${ }^{21,22}$. Esta forma de ensinar-aprender promove a ruptura do paradigma de que a aprendizagem teórica deve preceder a prática ${ }^{8}$.

Esta pesquisa apresenta algumas limitações, pois seus resultados não podem ser generalizados, por apresentar particularidades que envolvem docentes de um contexto regional e de instituição pública. Entretanto, apresenta subsídios para repensar a discussão do ensino da SAE, com intuito de aprimorar e sistematizar a assistência de enfermagem.

\section{CONSIDERAÇÕES FINAIS}

A SAE foi identificada como uma forma de respaldar o enfermeiro em suas ações, sendo imprescindível sua elaboração durante a prática de enfermagem, o que facilita e gerencia a assistência prestada ao indivíduo.

Os resultados deste estudo revelam que a temática SAE durante a graduação de enfermagem está em ascensão no campo científico, entretanto, ainda há dificuldades na implementação deste método durante a formação. Acredita-se que 0 ensino da SAE se estabelece sob influência de alguns fatores, dentre eles, as metodologias e estratégias de ensino, utilizados pelos docentes. $O$ ensino e aplicabilidade da SAE deve ser significativa para os alunos, pois influenciará na adesão e operacionalização futura, na condição de enfermeiro.

\section{REFERÊNCIAS}

1. Resolução COFEN n॰ 358 de 15 de outubro de 2009. Dispõe sobre a Sistematização da Assistência de Enfermagem - SAE - nas Instituições de Saúde Brasileiras. Rio de Janeiro: COFEN; 2009.

2. Alfaro-Lefevre R. Aplicação do Processo de Enfermagem: uma ferramenta para o pensamento crítico. Porto Alegre: Artmed; 2014.

3. Lei n. 7498, de 25 de junho de 1986. Dispõe sobre a regulamentação do exercício da enfermagem e dá outras providências. Diário Oficial da República Federativa do Brasil, Seção 1; p. 1. Brasília; 1986.

4. Soares MI, Resck ZMR, Terra FS, Camelo SHH. Sistematização da assistência de 
enfermagem: facilidades e desafios do enfermeiro na gerência da assistência. Esc Anna Nery. 2015; 19(1):47-53.

5. Fuly P SC, Leite JL, Lima SBS. Correntes de pensamento nacionais sobre sistematização da assistência de enfermagem. Rev Bras Enferm. 2008; 61(6):883-887.

6. Marinelli NP, Silva ARA, Silva DNO. Sistematização da Assistência de enfermagem: desafios para a implantação. Rev Enferm Contemporânea. 2015; 4(2):254-263.

7. Leadebal ODCP, Fontes WD, Silva CC. Ensino do processo de enfermagem: planejamento e inserção em matrizes curriculares. Rev Esc Enferm USP. 2010; 44(1):190-198.

8. Freitas DA, Santos EMS, Lima LVS, Miranda LN, Vasconcelos EL, Nagliate PC. Saberes docentes sobre o processo ensino-aprendizagem e sua importância para sua formação profissional em saúde. Interface (Botucatu). 2016; 20(57):437-448.

9. Silva AGI, Peixoto MAP, Brandão MAG, Ferreira MA, Martins JSA. Nursing students' difficulties in the learning of diagnostics in nursing, in a metacognitive perspective. Esc Anna Nery. 2011; 15(3):466-471.

10. Silva CC, Gelbcke FL, Meirelles BNS, Arruda C, Goulart S, Souza AlJ. O ensino da Sistematização da Assistência na perspectiva de professores e alunos. Rev Eletr Enf. 2011; 13(2):174-181.

11. Conceição VM, Araújo JS, Oliveira RAA, Zago MMF, Souza RF, Santana $M E$, et al. Percepções culturais de acadêmicos e enfermeiros sobre a Sistematização da Assistência de
Enfermagem. Rev enferm UFSM. 2014; $4(2): 378-388$.

12. Leal LA, Camelo SHH, Santos FC. O docente de administração em enfermagem: formação e competências profissionais. Rev Enferm UFPE on line. 2017; 11(6):2329-2338.

13. Minayo MCS. 0 desafio do conhecimento: pesquisa qualitativa em saúde. São Paulo: Hucitec; 2013.

14. Horr L, Gonçalves LHT, Saupe R. O ensino da metodologia assistencial de enfermagem. Rev Esc Enferm USP. 1987; 21(spe):40-54.

15. Menezes SRT, Priel MR, Pereira LL. Nurses autonomy and vulnerability in the Nursing Assistance Systematization practice. Rev Esc Enferm USP. 2011; 45(4):953-958.

16. Duran ECM, Toledo VP. Análise da produção do conhecimento em processo de enfermagem: estudo exploratório-descritivo. Rev Gaúch Enferm. 2011; 32(2):234-240.

17. Terra FS, Secco IAO, Robazzi MLCC. Perfil dos docentes de cursos de graduação em enfermagem de universidades públicas e privadas. Rev Enferm UERJ. 2011; 19(1):26-33.

18. Luzia MF, Costa FM, Lucena AF. O ensino das etapas do processo de enfermagem: revisão integrativa. Rev enferm UFPE on line. 2013; 7(esp):6678-6687.

19. Cogo ALP, Pedro ENR, Silveira DT, Silva APS, Alves RHK, Catalan VM. Desenvolvimento e utilização de objetos educacionais digitais no ensino de enfermagem. Rev Latino-Am enferm. 2007; 15(4):699-701. 
20. Tonhom SFR, Moraes MAA, Pinheiro OL. Formação de enfermeiros centrada na prática profissional: percepção de estudantes e professores. Rev Gaúch Enferm. 2016; 37(4):e63782.

21. Baldoino AS, Veras RM. Análise das atividades de integração ensinoserviço desenvolvidas nos cursos de saúde da Universidade Federal da Bahia. Rev Esc Enferm USP. 2016; 50(esp):17-24.
22. Tonhom SFR, Costa MCG, Hamamoto CG, Francisco AM, Moreira HM, Gomes R. A formação em enfermagem por área de competência: limites e possibilidades. Rev Esc Enferm USP. 2014; 48(Esp2):225-232.

Conflito de interesses: Os autores declaram não haver conflito de interesses.

\section{Participação dos autores:}

- Concepção: Rocha MMS, Mocheuti KN, Silvestre GCSB.

- Desenvolvimento: Rocha MMS, Mocheuti KN, Silvestre GCSB, Lima CM.

- Redação e revisão: Rocha MMS, Mocheuti KN, Silvestre GCSB, Lima CM, Ribeiro ADN.

Como citar este artigo: Rocha MMS, Mocheuti KN, Silvestre GCSB, Lima $C M$, Ribeiro ADN. Sistematização da assistência de enfermagem na perspectiva do docente. J Health NPEPS. 2019; 4(1):144-152. 